\title{
An explosive outbreak of Streptococcus pneumoniae serotype-8 infection in a highly vaccinated residential care home, England, summer 2012
}

\author{
H. L. THOMAS ${ }^{1,2} *$, R. GAJRAJ ${ }^{3}$, M.P. E. SLACK ${ }^{4}$, C. SHEPPARD ${ }^{4}$, \\ P. HAWKEY ${ }^{5,6}$, S. GOSSAIN ${ }^{5}$, C. M. DREW ${ }^{3}$ AND R. G. PEBODY ${ }^{1}$ \\ ${ }^{1}$ Centre for Infectious Disease Surveillance and Control, Public Health England, London, UK \\ ${ }^{2}$ UK Field Epidemiology Training Programme, Public Health England, London, UK \\ ${ }^{3}$ West Midlands Public Health England Centre, Birmingham, UK \\ ${ }^{4}$ Respiratory and Vaccine Preventable Bacteria Reference Unit, Public Health England, London, UK \\ ${ }^{5}$ Public Health England Birmingham Laboratory, Health of England NHS Foundation Trust, Birmingham, UK \\ ${ }^{6}$ Institute of Microbiology \& Infection, Biosciences, University of Birmingham, Birmingham, UK
}

Received 20 May 2014; Final revision 6 August 2014; Accepted 27 August 2014;

first published online 9 October 2014

\section{SUMMARY}

In August 2012, an explosive outbreak of severe lower respiratory tract infection (LRTI) due to Streptococcus pneumoniae serotype-8 occurred in a highly vaccinated elderly institutionalized population in England. Fifteen of 23 residents developed LRTI over 4 days (attack rate 65\%); 11 had confirmed $S$. pneumoniae serotype-8 disease, and two died. Following amoxicillin chemoprophylaxis and pneumococcal polysaccharide vaccine (PPV) re-vaccination no further cases occurred in the following 2 months. No association was found between being an outbreakassociated case and age $(P=0 \cdot 36)$, underlying comorbidities [relative risk (RR) $0 \cdot 8495 \%$ confidence interval (CI) 0.34-2.09], or prior receipt of PPV (RR 1·4, 95\% CI 0.60-3.33). However, the median number of years since PPV was significantly higher for cases $(n=15,10 \cdot 2$ years, range $7 \cdot 3-17 \cdot 9$ years) than non-cases $(n=8,7 \cdot 2$ years, range $6 \cdot 8-12 \cdot 8$ years) $(P=0 \cdot 045)$, provided evidence of waning immunity. Alternative vaccination strategies should be considered to prevent future $S$. pneumoniae outbreaks in institutionalized elderly populations.

Key words: Disease outbreaks, pneumococcal infections, pneumococcal polysaccharide vaccine.

\section{INTRODUCTION}

Streptococcus pneumoniae is a major cause of morbidity and mortality, with the burden of invasive pneumococcal disease (IPD) and pneumococcal pneumonia falling disproportionately on the elderly [1]. Although $S$. pneumoniae usually causes sporadic infections, outbreaks of severe pneumococcal disease caused by a single serotype are occasionally

\footnotetext{
* Author for correspondence: Dr H. L. Thomas, Centre for Infectious Disease Surveillance and Control, Public Health England, London, UK.

(Email: lucy.thomas@phe.gov.uk)
}

recognized, particularly in institutionalized settings. A systematic review of outbreaks of serious pneumococcal disease in closed settings [2] identified only 11 published outbreaks in elderly care residential settings over four decades [3-12], with clinical attack rates ranging from $3 \cdot 5-15 \%$. These outbreaks all occurred in settings with a low uptake of pneumococcal polysaccharide vaccine (PPV), and increasing PPV uptake was recommended to prevent future outbreaks.

Current vaccination strategies both in the USA [13] and the UK [14] recommend a single dose of the 23 -valent PPV to those aged $\geqslant 65$ years. We report an explosive outbreak of severe lower respiratory tract infection (LRTI) due to $S$. pneumoniae serotype- 8 
in a residential care home for the elderly in England in August 2012. Unlike previous reported outbreaks, the population had a high uptake of the PPV, which contains the serotype- 8 outbreak strain.

\section{Background and outbreak setting}

On 9 August 2012, staff of a residential home in England notified the Health Protection Agency (HPA) of an outbreak of acute respiratory tract infection among elderly residents. The residential home was a three-floor building housing 23 long-stay elderly residents, cared for by 22 staff. The residents each had their own individual rooms, apart from two couples, but mixed freely in social and dining areas.

Seven of the 23 residents had developed symptoms of cough and breathlessness since 8 August. One resident had been admitted to hospital; a chest radiograph showed right basal consolidation and $S$. pneumoniae was grown from blood culture. An outbreak of S. pneumoniae disease was suspected, and urine samples and sputum, when available, were collected from all symptomatic residents.

\section{Control measures}

On 9 August, the home was closed to new admissions and enhanced infection control measures were introduced, including isolation and cohorting of symptomatic residents, use of personal protective equipment, and enhanced cleaning.

By 11 August, three cases had been diagnosed with disease caused by S. pneumoniae (one bacteraemia, two urine antigen positive), establishing that an outbreak of pneumococcal disease had occurred. The outbreak-associated cases were treated with either amoxicillin or erythromycin as appropriate. Chemoprophylaxis (500 $\mathrm{mg}$ amoxicillin twice daily for 7 days) was given to all asymptomatic residents. On 21 August, PPV vaccination was given to all 13 residents who had not been vaccinated within the previous 10 years. Active follow-up for further cases continued for 2 months.

\section{METHODS}

\section{Outbreak investigation}

The study population was defined as all residents of the residential home during the period from 8 to 22 August 2012.

The following case definitions were used:
Probable case. Resident with a clinical diagnosis of LRTI with onset from 8 to 22 August 2012.

Confirmed case. Probable case with $S$. pneumoniae serotype- 8 identified in a blood culture or by urine antigen testing.

Outbreak-associated case. A confirmed or probable case.

\section{Microbiological methods}

Sputum was collected from symptomatic residents with productive cough and cultured on blood agar (with optochin disc), chocolate and CLED agar plates following homogenization of the sample using Sputasol (Oxoid, UK). Plates were incubated at $37^{\circ} \mathrm{C}$ in $\mathrm{CO}_{2}$ and examined for characteristic colonies after 18-24 h incubation. Any colonies on blood agar with typical morphology were confirmed as $S$. pneumoniae if they were susceptible to optochin. The $S$. pneumoniae isolated from the hospitalized resident's blood culture was confirmed using the above methods, and in addition was serotyped.

Nasopharyngeal swabs were collected from the symptomatic residents and tested against a PCR respiratory virus panel including influenza $\mathrm{A}$ and $\mathrm{B}$, parainfluenza virus, Adenovirus, Rhinovirus, respiratory syncytial virus (RSV) and human metapneumovirus, Urine samples were collected from symptomatic residents and tested using the BinaxNOW pneumococcal urinary antigen test (Binax, USA) and the Bio-Plex pneumococcal serotype assay (Bio-Rad, USA) to determine serotype [15]. In the case of one of the patients who died, and for whom a urine sample was not available, a nasopharyngeal swab was tested using the Binax NOW and Bio-Plex assays.

\section{Analytical epidemiological study}

We conducted a retrospective cohort study to investigate factors associated with being an outbreakassociated case during the outbreak period. The cohort included all residents of the home during the outbreak period.

Demographic data and information about symptoms were collected from the residential home manager. Further information on symptoms, and details of comorbidities and previous vaccination with PPV were obtained from the residents' general practitioners.

Data were analysed using Stata v. 12 (StataCorp., USA). Attack rates and relative risks were calculated for all binary variables; Fisher's exact $P$ values were 


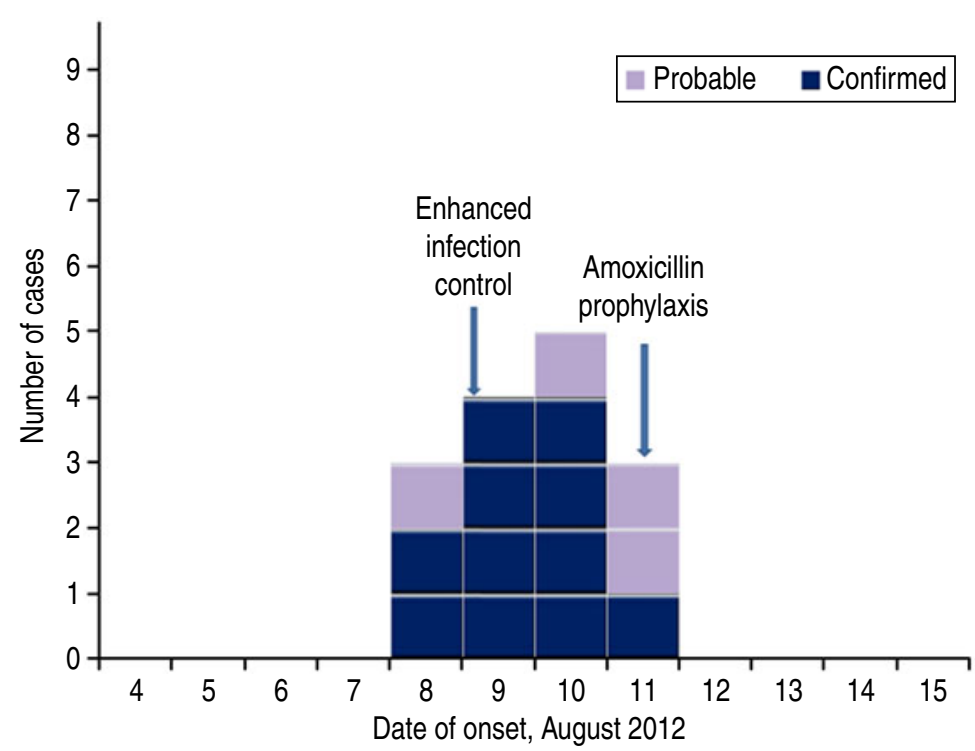

Fig. 1. Distribution of cases of lower respiratory tract infection by date of onset, residential home, England, August 2012.

calculated due to small sample size. The distribution of continuous variables was compared using the Mann-Whitney rank sum test.

\section{RESULTS}

\section{Descriptive epidemiology}

The median age of the 23 residents was 90 years (range 75-94 years) and 15/23 were female. All but three had underlying comorbidities.

There were 15 outbreak-associated cases. All 15 had symptoms of cough; in addition, five reported anorexia, three had wheeze and two had shortness of breath. Only one case was febrile, and a blood sample for culture was collected for this patient. Eleven cases were microbiologically confirmed, one by positive blood culture and the remaining ten cases by urinary antigen testing. This gave a confirmed-case attack rate of $48 \%$. An additional four probable cases also occurred during the outbreak period (outbreak-associated case attack rate $65 \%$ ). Two of the outbreak-associated cases died (case-fatality ratio $13 \%$ ).

Eight $(73 \%)$ of the 11 confirmed cases had previously been vaccinated with PPV, a median of 12 years prior to the outbreak (range 8-18 years). Twelve $(80 \%)$ of the 15 outbreak-associated cases had previously been vaccinated with PPV, a median of 10 years prior to the outbreak (range 7-18 years).

None of the residents had had symptoms typical of a viral respiratory infection prior to the outbreak. No staff were symptomatic during the outbreak period.
The distribution of cases by date of onset is shown in Figure 1. All cases clustered over the 4 days from 8 to 11 August, with no new cases occurring in the subsequent 2 months' follow-up.

\section{Microbiological findings}

No pathogenic bacteria were isolated from sputum of the three residents who had a productive cough. The $S$. pneumoniae isolated from the blood culture was found to be serotype-8, MLST sequence type 53, and susceptible to penicillin, erythromycin and tetracycline.

The microbiological results for the 15 outbreakassociated cases are presented in Table 1 .

\section{Analytical epidemiology}

The clinical attack rates by key epidemiological characteristics are presented in Table 2. The attack rate was very similar in males and females [relative risk (RR) 0.94, 95\% confidence interval (CI) $0 \cdot 49$ 1.79]. The median age of the outbreak-associated cases ( 89 years, range $77-93$ years) was very similar to the median age of the non-cases (91 years, range 7594 years, $P=0 \cdot 36$ ). There was no significant association between being a outbreak-associated case and the presence of any underlying comorbidities $(P=1 \cdot 0)$.

There was no association between prior receipt of PPV and being an outbreak-associated case (RR 1.4, 95\% CI $0 \cdot 60-3 \cdot 33, P=1 \cdot 0$ ). However, the median 
Table 1. Laboratory results of outbreak-associated cases of lower respiratory tract infection in a residential home, England, 2012

\begin{tabular}{|c|c|c|c|c|c|}
\hline Case & Blood culture & $\begin{array}{l}\text { Nasal swab } \\
\text { PCR respiratory } \\
\text { virus panel }\end{array}$ & $\begin{array}{l}\text { Binax assay on } \\
\text { urine }(\mathrm{U}) \text { or nasal } \\
\text { swab }(\mathrm{N})\end{array}$ & $\begin{array}{l}\text { Bio-plex assay } \\
\text { on urine }(\mathrm{U}) \text { or } \\
\text { nasal swab }(\mathrm{N})\end{array}$ & $\begin{array}{l}\text { Clinical } \\
\text { outcome }\end{array}$ \\
\hline 1 & $\begin{array}{l}\text { S. pneumoniae } \\
\text { serotype-8 }\end{array}$ & & & & Died \\
\hline 2 & & Negative & & & Survived \\
\hline 3 & & Negative & Negative (U) & S. pneumoniae serotype $8(\mathrm{U})$ & Survived \\
\hline 4 & & Negative & Negative (U) & S. pneumoniae serotype 8 (U) & Survived \\
\hline 5 & & Negative & Negative (U) & S. pneumoniae serotype $8(\mathrm{U})$ & Survived \\
\hline 6 & & Negative & Negative (U) & S. pneumoniae serotype $8(\mathrm{U})$ & Survived \\
\hline 7 & & Rhinovirus & Positive $(\mathrm{N})$ & S. pneumoniae serotype $8(\mathrm{~N})$ & Died \\
\hline 8 & & Negative & Positive (U) & S. pneumoniae serotype $8(\mathrm{U})$ & Survived \\
\hline 9 & & & Positive (U) & S. pneumoniae serotype $8(\mathrm{U})$ & Survived \\
\hline 10 & & & Positive (U) & S. pneumoniae serotype $6 \mathrm{~A} / \mathrm{C}(\mathrm{U})$ & Survived \\
\hline 11 & & & Negative (U) & S. pneumoniae serotype $8(\mathrm{U})$ & Survived \\
\hline 12 & & & Negative (U) & S. pneumoniae serotype $8(\mathrm{U})$ & Survived \\
\hline 13 & & & Negative (U) & Not detected & Survived \\
\hline 14 & & & Negative (U) & Not detected & Survived \\
\hline 15 & & & Negative (U) & S. pneumoniae serotype $8(\mathrm{U})$ & Survived \\
\hline
\end{tabular}

number of years since receipt of PPV was significantly higher for outbreak-associated cases $(10 \cdot 2$ years, range $7 \cdot 3-17 \cdot 9$ years $)$ compared to non-cases $(7 \cdot 2$ years, range $6 \cdot 8-12 \cdot 8$ years), Wilcoxon rank sum $P=0 \cdot 045$.

A sensitivity analysis was conducted restricting the analysis to the confirmed cases $(n=11)$ and non-cases $(n=8)$. The results obtained were very similar. Again, there was no association between being a case and prior receipt of PPV (RR 1.23, 95\% CI 0.50-3.05, $P=1 \cdot 0$ ), and the median number of years since receipt of PPV was significantly higher for the cases $(11.7$ years, range $8 \cdot 0-17.9$ years) compared to the noncases $(7 \cdot 2$ years, range $6 \cdot 8-12 \cdot 8$ years, $P=0 \cdot 040)$.

\section{DISCUSSION}

This was an unusual outbreak of LRTI due to $S$. pneumoniae serotype-8 with an exceptionally high attack rate during summertime.

Prompt reporting of the outbreak led to rapid identification of the causative agent and implementation of control measures. No new outbreak-associated cases developed over the 2-month period since antibiotic chemoprophylaxis of asymptomatic residents and re-vaccination.

The exceptionally high attack rate in a population with a high uptake of PPV-23, which includes the serotype- 8 antigen, is of particular concern. Previously published outbreaks of $S$. pneumoniae in residential facilities for the elderly have all reported low levels of PPV vaccination. In this outbreak, $80 \%$ of outbreakassociated cases had documented evidence of PPV vaccination. The finding that all the vaccinated cases had received PPV more than 7 years prior to the outbreak, and that cases had received vaccine significantly longer ago than non-cases, suggests that the low level of PPV protection may in part reflect waning immunity.

A small number of published observational studies have found a low level of PPV vaccine effectiveness against IPD in the elderly, and a decrease in effectiveness after 5 years [16, 17]. However, although there is evidence that vaccine effectiveness varies by serotype [18], these studies have not had the power to estimate serotype-specific vaccine effectiveness by time since vaccination. Evidence of the effectiveness of PPV against pneumonia in the elderly is even sparser, with the few published studies to date providing contradictory findings $[19,20]$. This could in part be explained by lack of consideration of time since vaccination as an important effect modifier. Waning vaccine effectiveness is consistent with what is known about the immune response to PPV, as PPV-induced capsular-specific antibody levels have been shown to decrease over time [21]. Our findings provide evidence of waning immunity of PPV in the prevention of severe LRTI due to serotype- 8 disease in an elderly population. Current vaccination strategies both in the USA [13] and the UK [14] recommend only a 
Table 2. Attack rates and relative risks for outbreak-associated cases (probable and confirmed cases) $(n=15)$ in residents of a residential home, England, 2012 by potential risk factors $(N=23)$

\begin{tabular}{|c|c|c|c|c|c|c|c|}
\hline \multirow[b]{2}{*}{ Characteristics } & & \multicolumn{3}{|c|}{$\begin{array}{l}\text { Attack rate among } \\
\text { exposed }\end{array}$} & \multirow[b]{2}{*}{ RR } & \multirow[b]{2}{*}{$95 \% \mathrm{CI}$} & \multirow{2}{*}{$\begin{array}{l}\text { Fisher's exact } \\
P \text { value }\end{array}$} \\
\hline & & No. & Total & $\%$ & & & \\
\hline \multirow[t]{2}{*}{ Sex } & Female & 5 & 8 & $62 \cdot 5$ & $0 \cdot 94$ & $0 \cdot 49-1 \cdot 79$ & $1 \cdot 0$ \\
\hline & Male & 10 & 15 & $66 \cdot 7$ & & & \\
\hline \multirow[t]{2}{*}{ Age } & $<90$ years & 8 & 11 & 50 & $0 \cdot 8$ & $0 \cdot 44-1 \cdot 46$ & $0 \cdot 8$ \\
\hline & $\geqslant 90$ years & 7 & 12 & $58 \cdot 3$ & & & \\
\hline \multirow[t]{3}{*}{ Floor } & 2 & 2 & 5 & 40 & Ref. & & $0 \cdot 33$ \\
\hline & 1 & 7 & 11 & $63 \cdot 6$ & $1 \cdot 59$ & $0 \cdot 5-5 \cdot 09$ & \\
\hline & $\mathrm{G}$ & 6 & 7 & $85 \cdot 7$ & $2 \cdot 14$ & $0 \cdot 7-6 \cdot 5$ & \\
\hline \multirow[t]{16}{*}{ Comorbidities } & Chronic heart disease: Yes & 6 & 7 & $85 \cdot 7$ & $1 \cdot 52$ & $0 \cdot 9-2 \cdot 58$ & $0 \cdot 35$ \\
\hline & Chronic heart disease: No & 9 & 16 & $56 \cdot 3$ & & & \\
\hline & Chronic resp. disease: Yes & 2 & 2 & 100 & $1 \cdot 62$ & $1 \cdot 15-2 \cdot 26$ & $0 \cdot 53$ \\
\hline & Chronic resp. disease: No & 13 & 21 & $61 \cdot 9$ & & & \\
\hline & Diabetes: Yes & 2 & 4 & 50 & $0 \cdot 73$ & $0 \cdot 26-2 \cdot 04$ & $0 \cdot 59$ \\
\hline & Diabetes: No & 13 & 19 & $68 \cdot 4$ & & & \\
\hline & Chronic renal disease: Yes & 6 & 9 & $66 \cdot 7$ & $1 \cdot 04$ & $0 \cdot 57-1 \cdot 9$ & $1 \cdot 0$ \\
\hline & Chronic renal disease: No & 9 & 14 & $64 \cdot 3$ & & & \\
\hline & Cardiovascular disease: Yes & 2 & 5 & 40 & $0 \cdot 55$ & $0 \cdot 18-1 \cdot 68$ & $0 \cdot 3$ \\
\hline & Cardiovascular disease: No & 13 & 18 & 72 & & & \\
\hline & Hypothyroidism: Yes & 2 & 3 & $66 \cdot 7$ & $1 \cdot 03$ & $0 \cdot 43-2 \cdot 43$ & $1 \cdot 0$ \\
\hline & Hypothyroidism: No & 13 & 20 & 65 & & & \\
\hline & Dementia: Yes & 2 & 3 & $66 \cdot 7$ & $1 \cdot 03$ & $0 \cdot 43-2 \cdot 43$ & $1 \cdot 0$ \\
\hline & Dementia: No & 13 & 20 & 65 & & & \\
\hline & Any comorbidities: Yes & 9 & 16 & $56 \cdot 3$ & $0 \cdot 84$ & $0 \cdot 34-2 \cdot 09$ & $1 \cdot 0$ \\
\hline & Any comorbidities: No & 2 & 3 & $66 \cdot 7$ & & & \\
\hline \multirow[t]{2}{*}{ PPV } & Yes & 12 & 17 & $70 \cdot 6$ & $1 \cdot 41$ & $0 \cdot 60-3 \cdot 33$ & $1 \cdot 0$ \\
\hline & No & 3 & 6 & 50 & & & \\
\hline \multirow{4}{*}{$\begin{array}{l}\text { Among those vaccinated, } \\
\text { years since PPV }\end{array}$} & $\leqslant 5$ & 0 & 0 & 0 & n.a. & & $0 \cdot 61$ \\
\hline & $6-10$ & 6 & 10 & 60 & Ref. & & \\
\hline & $11-15$ & 4 & 5 & 80 & $1 \cdot 3$ & $0 \cdot 68-2 \cdot 60$ & \\
\hline & $15-20$ years & 2 & 2 & 100 & $1 \cdot 7$ & $1 \cdot 05-2 \cdot 89$ & \\
\hline
\end{tabular}

RR, Relative risk; CI, confidence interval; n.a., not applicable; PPV, pneumococcal polysaccharide vaccine.

single dose of PPV to those aged $\geqslant 65$ years. If this is given at around the age of 70 , more than 10 years will have elapsed by the time such individuals are in their 80 s and 90 s and at greatest risk of requiring institutionalized care.

The fact that this particularly aggressive outbreak was caused by $S$. pneumoniae serotype-8, a highly invasive strain with a very high case-to-carrier ratio [22], is of concern. To date serotype- 8 has not been one of the main causes of IPD in the UK [23], and there has only been one previously reported small institutional outbreak due to this serotype [24]. However, the number of reports of IPD due to serotype-8 in those aged $\geqslant 65$ years has increased by $15 \%$ from 2003-2006 to 2009-2011 (PHE, unpublished data). This could reflect natural secular fluctuations in serotype- 8 disease [25]. Alternatively, this trend could be a response to the introduction of the pneumococcal conjugate vaccines PCV-7 and PCV-13, which do not contain serotype- 8 , into the childhood vaccination programme in the UK in 2007 and 2010, respectively. While there is clear evidence of serotype replacement following the introduction of both PCV-7 and PCV-13 in the UK, it is not yet clear whether this is leading to an overall increase in carriage of serotypes with a high case-to-carrier ratio such as serotype- 8 and $22 \mathrm{~F}$, which are more virulent and could lead to more frequent outbreaks of highly invasive strains [26]. Further studies of population carriage of different $S$. pneumoniae serotypes since the introduction of $\mathrm{PCV}-13$ are required.

The detection of this outbreak was possible due to Binax testing in the local laboratory to identify cases of pneumococcal disease, followed by the use of a 
specialist serotype-specific Bio-Plex multiplex antigen detection assay. This assay has been shown to be $79 \cdot 3 \%$ sensitive and $99 \cdot 3 \%$ specific in validation experiments on clinical urine specimens from patients with matching blood culture of either pneumococcus or other organisms [15]. However, in this outbreak several urine specimens that were negative in the BinaxNOW test, were positive for serotype-specific pneumococcal antigen. This is in line with a recent large study on community-acquired pneumonia, in which 144 patients with BinaxNOW-negative results gave a serotype in the Bio-plex assay [27]. This data, and analytical data from samples spiked with antigens (C. Sheppard, unpublished data), suggest that the multiplex assay is capable of detecting antigen at a much lower level than the Binax assay. Greater use of the Bio-plex assay in cases of suspected pneumococcal disease would appear to be warranted to increase sensitivity and identify the relevant serotype.

The presence of a single clinical case positive for $S$. pneumoniae serotype $6 \mathrm{~A} / \mathrm{C}$ in an outbreak of serotype- 8 is interesting. While this case could represent a coincidental case of infection with a nonoutbreak serotype, a non-specific cross-reaction between serotypes cannot be ruled out, although the Bio-plex assay has previously been shown to have high serotype specificity $(99 \cdot 3 \%)$ [15]. Another alternative explanation is that this represents the detection of antigen from carriage in an elderly patient, as specific data on serotype carriage and associated multiplex urine specimen tests on elderly patients do not exist. However, this patient had clinical symptoms compatible with $S$. pneumoniae infection, and was also BinaxNOW positive, suggesting that this result was not obtained purely by the enhanced sensitivity of the Bio-Plex assay.

Another unusual aspect of this outbreak was that it occurred in the summer, and there was no evidence of any preceding viral respiratory infections in residents.

This study has a number of limitations. In this very rapidly evolving outbreak, the need to take public health action took precedence over the analytical study, so it was not possible to take pre- and postchemoprophylaxis nasopharyngeal swabs on asymptomatic residents or staff. It was therefore not possible to describe the extent of asymptomatic carriage of the outbreak strain in the population of the residential home, or to evaluate the impact of amoxicillin chemoprophylaxis on carriage. We were therefore not able to disentangle the possible impact of exhaustion of susceptibles, chemoprophylaxis, infection control measures and PPV in halting the progression of the outbreak, although the latter would have taken 10-14 days to have an effect. No data were collected on the smoking status of residents, so it was not possible to analyse whether smoking was a risk factor or confounder in this outbreak. In the UK, PPV vaccination is not recommended for residential or nursing staff without individual clinical risk factors warranting vaccination, so details of PPV vaccination status of staff were not collected.

This outbreak highlights the importance of early reporting and investigation of clusters of severe respiratory illness in care homes for the elderly all year round, using both culture- and non-culture-based techniques, to enable prompt initiation of control measures. The very high attack rate despite a high level of previous PPV vaccination, and the fact that cases had a longer time since vaccination than non-cases, adds to the small body of evidence that PPV is of only limited effectiveness in preventing pneumococcal pneumonia in the elderly, and that effectiveness wanes over time. This highlights the importance of considering the diagnosis of pneumococcal pneumonia/IPD even in a highly vaccinated population. Furthermore, this outbreak reinforces the need to improve the evidence base on alternative vaccination strategies, including studies on the efficacy of pneumococcal conjugate vaccines in the elderly, although none of the current conjugate vaccines protect against serotype- 8 and so would not have provided protection in this particular outbreak. Finally, further work is required on the indirect impact of the universal childhood PCV-13 vaccination programme, including serotype replacement, on serotypespecific pneumococcal pneumonia and IPD in this high-risk population.

\section{ACKNOWLEDGEMENTS}

The authors thank the residential home staff and general practitioners for their role in the rapid response to this outbreak and Dr M. Weinbren for forwarding the $S$. pneumoniae isolate. The authors also thank Nick Andrews, PHE, for commenting on the statistical analysis and Elizabeth Miller, PHE, for supplying background information on the trends of $S$. pneumoniae serotype- 8 disease in the UK.

\section{DECLARATION OF INTEREST}

P.M.H. has received research funding and/or consultancy payments from bioMérieux, BectonDickinson, Eumedica, Merck, Novartis, Magus Communications, Pfizer and Wyeth. P.M.H. is the 
director of ModusMedica (a medical education company). M.P.E.S. has received research funding and/ or support to attend scientific conferences from Pfizer, GSK and MSD. C.S. has received support to attend conferences from Pfizer and Wyeth. The other authors have no interests to declare.

\section{REFERENCES}

1. Melegaro A, et al. The current burden of pneumococcal disease in England and Wales. Journal of Infection 2006; 52: $37-48$.

2. Ihekweazu C, et al. Outbreaks of serious pneumococcal disease in closed settings in the post-antibiotic era: a systematic review. Journal of Infection 2010; 61: 21-27.

3. Quick RE, et al. Underutilisation of pneumococcal vaccine in nursing home in Washington State: report of a serotype-specific outbreak and a survey. American Journal of Medicine 1993; 94: 149-152.

4. Olver WJ, et al. Investigation and control of penicillin non-susceptible Streptococcus pneumoniae infections in a care home. Journal of Hospital Infection 2008; 26: 248-255.

5. Tan CG, Ostrawski S, Bresnitz EA. A preventable outbreak of pneumococcal pneumonia among unvaccinated nursing home residents in New Jersey during 2001. Infection Control and Hospital Epidemiology 2003; 24: 848-852.

6. Gleich S, et al. Streptococcus pneumoniae serotype 4 outbreak in a home for the aged: report and review of recent outbreaks. Infection Control and Hospital Epidemiology 2000; 21: 711-717.

7. Hansmann Y, et al. An outbreak of pneumococcal pneumonia among residents of a retirement home in France during October 2003. Infection Control and Hospital Epidemiology 2006; 27: 1252-1254.

8. Nuorti JP, et al. An outbreak of multidrug-resistant pneumococcal pneumonia and bacteraemia among unvaccinated nursing home residents. New England Journal of Medicine 1998; 338: 1861-1868.

9. Centres for Disease Control and Prevention. Outbreaks of pneumococcal pneumonia among unvaccinated residents in chronic care facilities-Massachusetts, October 1995, Oklahoma February 1996 and Maryland May-June 1996. Morbidity and Mortality Weekly Report 1997; 46: 60-62.

10. Centers for Disease Control and Prevention. Outbreak of pneumococcal pneumonia among unvaccinated residents of a nursing home-New Jersey-April 2001. Journal of the American Medical Association 2001: 286: $1570-1571$.

11. Sheppard DC, Bartlett KA, Lampiris HW. Streptococcus pneumoniae transmission in chronic-care facilities: description of an outbreak and review of management strategies. Infection Control and Hospital Epidemiology 1998; 19: 851-853.

12. Fiore AE, et al. Outbreak of pneumonia in a long-term care facility: antecedent human parainfluenza virus 1 infection may predispose to bacterial pneumonia. Journal of the American Geriatric Society 1998; 46: 1112e7.
13. Centers for Disease Control and Prevention. Epidemiology and Prevention of Vaccine-Preventable Diseases, 12th edn. May 2012 (http://www.cdc.gov/vaccines/pubs/ pinkbook/pneumo.html).

14. Department of Health. Immunisation against infectious disease. Updated 2012. (https://www.wp.dh.gov.uk/immunisation/files/2012/10/Green-Book-Chapter-25-v4_0. pdf.pdf).

15. Sheppard CL, et al. Development of a sensitive, multiplexed immunoassay using xMAP beads for detection of serotype-specific streptococcus pneumoniae antigen in urine samples. Journal of Medical Microbiology 2011; 60: 49-55.

16. Andrews NJ, et al. Impact and effectivenesss of 23-valent pneumococcal polysaccharide vaccine against invasive pneumococcal disease in the elderly in England and Wales. Vaccine 2012; 30: 6802-6808.

17. Shapiro ED, et al. The protective efficacy of polyvalent pneumococcal polysaccharide vaccine. New England Journal of Medicine 1991; 325: 1453-1460.

18. Andrews NJ, et al. Impact and effectivenesss of 23 -valent pneumococcal polysaccharide vaccine against invasive pneumococcal disease in the elderly in England and Wales. Vaccine 2012; 30: 6802-6808.

19. Orqvist A, et al. and Swedish Pnumococcal Vaccination Study Group. Randomised trial of 23-valent pneumococcal capsular polysaccharide vaccine in prevention of pneumonia in middle-aged and elderly people. Lancet 1998; 351: 399-403.

20. Maruyama T, et al. Efficacy of 23-valent pneumococcal vaccine in preventing pneumonia in improving survival in nursing home residents: double blind, randomised and placebo controlled trial. British Medical Journal 2010; 340: c1004.

21. Artz As, Ershler WB, Longon DL. Pneumococcal vaccination and revaccination in older adults. Clinical and Microbiological Review 2003; 16: 308-318.

22. Miller E, et al. Herd immunity and serotype replacement 4 years after seven-valent pneumococcal conjugate vaccination in England and Wales: an observational cohort study. Lancet Infectious Diseases 2011; 11: 760-768.

23. Flashce S, et al. Effect of pneumoccccal vaccination on serotype-specific carriage and invasive disease in England: a cross-sectional study. PLoS Medicine 2011; 8: e1001017.

24. Birtles A, et al. Multilocus sequence typing directly on DNA from clinical samples and a cultured isolate to investigate linked fatal pneumococcal disease in residents of a shelter for homeless men. Journal of Clinical Microbiology 2005; 43: 2004-2008.

25. Harboe ZB, et al. Temporal trends in invasive pneumococcal disease and pneumococcal serotypes over $7 \mathrm{dec}$ ades. Clinical Infectious Diseases 2010; 50: 329-337.

26. Miller E, et al. Herd immunity and serotype replacement 4 years after seven-valent pneumococcal conjugate vaccination in England and Wales: an observational cohort study. Lancet Infectious Diseases 2011; 11: 760-768.

27. Bewick T, et al. Serotype prevalence in adults hospitalised with pneumococcal non-invasive community-acquired pneumonia. Thorax 2012; 67: 540-545. 